Dom. Cien., ISSN: 2477-8818

Vol. 3, núm. 4, octubre, 2017, pp. 36-46

\title{
Incompatibilidad Rh en el embarazo
}

\section{Rh incompatibility in pregnancy}

\section{Incompatibilidade $\mathbf{R h}$ na gravidez}

\author{
Carlos A. Vizueta-Chávez ${ }^{\text {I }}$ \\ carlos.vizuetac@ug.edu.ec \\ Byron O. López Silva II \\ byron.lopezs@ug.edu.ec \\ Jorge E. Balon-Benavides III \\ jorgebb@gamil.com \\ Rosario H. Zambrano-Bonilla IV \\ rosario.hz@gmail.com
}

Recibido: 30 de enero de 2017 * Corregido: 20 de febrero de 2017 * Aceptado: 20 junio de 2017

\footnotetext{
I. Médico, Universidad de Guayaquil, Guayaquil, Ecuador. Médico, Universidad de Guayaquil, Guayaquil, Ecuador. Médico, Universidad de Guayaquil, Guayaquil, Ecuador.

Iv. Médico, Universidad de Guayaquil, Guayaquil, Ecuador.
} 


\title{
Resumen
}

$\mathrm{La}$ incompatibilidad $\mathrm{Rh}$ en el embarazo se presenta en paciente $\mathrm{Rh}$ negativo cuyo padre es $\mathrm{Rh}$ positivo dando en el producto un trastorno de incompatibilidad sanguínea que ocasiona una enfermedad hemolítica en el feto. Es una afección inmunológica aloinmune donde anticuerpos maternos atacan la membrana de los eritrocitos fetales produciendo hemolisis.

Se presenta el caso de una paciente $\mathrm{Rh}(-)$ secundigesta de 31 años de edad, primer embarazo con evolución normal y con prevención de la vacuna anti-D cuya evolución llego a su feliz término teniendo su test de Coombs negativo y con la vacunación consiguiente. En su segunda gestación a pesar de la vacunación se produce la sensibilización a las 30 a 31 semanas aproximadamente con la sintomatología correspondiente, llegando hasta las 35 semanas gestacionales, siendo el momento apropiado de su extracción.

Palabras clave: Hemolisis; aloinmunización; isoinmunizacion; sensibilización.

\begin{abstract}
$\mathrm{Rh}$ incompatibility in pregnancy occurs in a $\mathrm{Rh}$ negative patient whose father is $\mathrm{Rh}$ positive giving the product a blood incompatibility disorder that causes hemolytic disease in the fetus. It is an alloimmune immunological condition where maternal antibodies attack the fetal erythrocyte membrane causing haemolysis.

The case of a 31-year-old female Rh (-) secundigesta, the first pregnancy with normal evolution and with the prevention of the anti-D vaccine, whose evolution came to a happy conclusion with her negative Coombs test and subsequent vaccination. In the second gestation, in spite of the vaccination, the sensitization takes place at approximately 30 to 31 weeks with the corresponding symptomatology, reaching up to 35 gestational weeks, being the appropriate moment of its extraction.
\end{abstract}

Key words: Hemolysis; alloimmunization; isoimmunization; sensitization. 


\section{Introducción.}

La enfermedad hemolítica del recién nacido o eritroblastosis fetal en un trastorno clínico obstétrico y neonatal, cuya prevalencia morbimortalidad ha disminuido gracias a la gran prevención empleada en pacientes susceptibles y sus tratamiento; igual esta una de las principales causas de muerte neonatal. Y cuya incidencia es aproximadamente un caso por cada 1000 nacidos vivos. ${ }^{1-3}$

La siguiente revisión intenta otorgar información básica, clínica, diagnostica y tratamiento con relación a un caso clínico en la cual se realizó seguimiento, medidas diagnosticas específicas y las razones de viabilidad que nos indicó realizar la culminación de la gesta. ${ }^{4}$

Lo importante es la prevención antes de un evento sensibilizante, ya que al estar sensibilizada ya se habrá formado los anticuerpos anti-D y el tratamiento base que es el suero anti-D no sería viable. Estos acontecimientos sensibilizante pueden ser ocasionados por parto o cesaría de un feto $\mathrm{Rh}+$, aborto, embarazo ectópico, traumatismo abdominal materno, hemorragias ante-parto en gestantes $\mathrm{Rh}-$ no sensibilizadas, etc. Es importante la administración de una dosis intramuscular de 300ug de gammaglobulina anti-d en las primeras 72 horas del proceso sensibilizante, es útil esta prevención hasta 4 semana post sensibilización. La causa más común de sensibilización es en el momento de separación en el parto (desprendimiento placentario) por lo cual se administra una dosis de anti-D (1500 UI) en las 72 horas antes del parto. ${ }^{5-7}$ 


\section{Clínica}

La incompatibilidad $\mathrm{Rh}$ produce la enfermedad hemolítica fetal o neonatal, resultado de la gran hemolisis y posterior compensación fetal. Dependiente del grado de hemolisis varía su forma de presentación, desde una forma leve que cursa con ictericia la cual se resuelve bien con luminoterapia, hasta una forma grave.

El resultado de la hemolisis y del secuestro eritrocitario son 2: la anemia hemolítica que es la base del cuadro en el feto y la hiperbilirrubinemia con predominio indirecta, que afecta al feto pero principalmente al recién nacido.

El feto se ve afectado principalmente por la anemia hemolítica que depende de la capacidad de la medula osea en producir hematíes. El hígado se encarga exclusivamente de la eritropoyesis produciendo la disminución de la síntesis de albumina, produciendo hipoalbuminemia que causa un descenso en la presión oncótica que lleva a desarrollar hydrops y anasarca.

A nivel cardiaco la hemolisis produce una reducción en el transporte de oxigeno ya que su principal transporte es el eritrocito, esta hipoxia produce al aumento de la frecuencia cardiaca intentando compensarla, si no se compensa termina produciendo insuficiencia cardiaca congestiva.

La hiperbilirrubinemia se debe a la incapacidad del recién nacido de eliminarlo, ya que va a ser excretado en forma conjugada con el ácido glucurónico cuya unión se realiza en el hígado el cual esta disminuido en el recién nacido y prematuro. La bilirrubina circula en sangre unida a albumina, su disminución produce que aumente la circulación de bilirrubina libre cuya acumulación termina en los tejidos nerviosos causando kernycterus y signos de disfunción cerebral como letardo, hipertonía y desaparición del signo de moro. 


\section{Diagnóstico}

El diagnóstico de la afección fetal por conflicto Rh(-) se debe de dar de forma progresiva, viendo la clínica de la paciente y los exámenes complementarios, siendo como enfoque principal el número de gesta y la detección del anticuerpo anti-D. Ante un progenitor $\mathrm{RH}(+)$ se investiga la presencia de anticuerpo Rh materno por medio del test de Coombs indirecto para determinar si se encuentra o no sensibilizada.

Test de Coombs indirecto negativo: seguimiento mensual hasta la semana 28, después con controles quincenales hasta el parto.

Lo importante es la detección temprana de afecciones fetales mediante un intenso control, realizándose titulación de anticuerpo cada 21 días. La gravedad de la anemia fetal se calcula a través de la concentración de bilirrubina en L.A, cuyo procedimiento resulta invasivo aumentando la probabilidad de sensibilización y el incremento de formación de anticuerpos por la madre. Para evitar esta agresión y evaluar el grado de anemia fetal se implementa la velocidad sistólica máxima de la arteria cerebral media mediante ecografía doopler detectando la bilirrubina por medio de densidad óptica a $450 \mathrm{NM}$ (valor delta OD450).

El estudio doopler se utiliza como principal método de control y seguimiento fetal en gestaciones de alto riesgo por isoinmunizacion. En la cual se va a investigar: velocidad sistólica en la arteria cerebral media (VS-ACM), presencia de hydrops y la cantidad de L.A.

La VS-ACM se relaciona, los rangos de la edad gestacional, con mayor VSM incrementa el riesgo de anemia fetal aumentan. Ante un valor mayor a 1.5 MoM se realizará 
la confirmación a las 24 horas. Se realizara VS-ACM en todo paciente en el cual el test de Coombs indirecto salga positivo para establecer anemia fetal severa, la medición puede iniciar a partir de las 18 semanas de gestación repitiéndose con un intervalo de 1 a 2 semanas. Si se encuentra excediendo los 1.5 MoM antes de la semana 24 se realizara cordocentesis para medir los niveles de hematocrito y ver la necesidad de realizar una transfusión intrauterina siendo esta invasiva pero necesaria.

El diagnostico ecográfico nos ayuda a observa la evolución de la anemia mediante determinantes que se encuentran en el feto, el L.A y la placenta; en fases tempranas inicia con; ascitis, derrame pericárdico, hepatoesplenomegalia, edema cutáneo y poli hidramnios, todos encontrándose en grado leve. En fase tardía además del empeoramiento de lo descrito, se puede observar la presencia de hydrops, la anasarca aparece en los fetos con $\mathrm{Hb}$ en niveles entre 2 a $6 \mathrm{~g} / \mathrm{dl}$.

\section{Profilaxis}

El suero de Rh inmunoglobulina D (anti D) se lo obtiene como producto de sangre humana obtenida de un pequeño grupo de donantes inmunizados.

Existen diferentes planos de administración preventiva según la región, no solo en la dosis administrada de anti-D sino también del cálculo cuantificativo en el volumen de la hemorragia materno fetal. En Australia, Reino Unido y Estados Unidos, la dosis es de 600 UI, 500UI y 1500 UI, respectivamente. Esta dosis cubre hemorragias de 6.5 y $15 \mathrm{ml}$ de glóbulos rojos Rh D positivo. En hemorragias mayores se adiciona anti-D si es necesario. En Alemania, y en otros países europeos así como en regiones latinas se administra una gran dosis única de 1500 UI de anti-D después de la semana 28 y otra dosis de 1500 UI las 72 horas previas al parto. Así también se necesita una dosis 
tras cualquier acontecimiento que pueda causar sensibilización como aborto, embarazo ectópico, trauma materno y cualquier procedimiento intrauterino invasivo.

La profilaxis anti-D, ya sea prenatal o postparto, solo puede suprimir la inmunización $\mathrm{Rh}$ primaria, no presenta respuesta en mujeres sensibilizadas que ya han desarrollado anticuerpos anti-D. Cerca de un 55\% de mujeres se sensibilizan antes del primer embarazo o ante algún evento que impulse la producción de IG anti-D, este fenómeno se conoce como "sensibilización silenciosa".

En las gestantes Rh-D negativas se determinaran los anticuerpos irregulares cada trimestre (10, 24 y 34-36 semanas), nunca en el periodo de un mes post-administración de gammaglobulina. Y si las titulaciones son $>1: 16$ se las considerara de alto riesgo y los controles serán a las 10, 17, 24, 29, 34 semanas.

Sin la aplicación de la inmunoprofilaxis en $10 \%$ de la madres Rh (D) negativas se sensibilizan en el primer embarazo, 30\% en el segundo y 50\% a partir del tercero. Aquí la importancia de controles prenatales y postparto ya que es una de las causas más importantes de morbimortalidad neonatal.

\section{Tratamiento}

En pacientes cuya inmunoprofilaxis no se realizó adecuadamente o a pesar de la realización apropiada existió una sensibilización con un test de Coombs positivo e identificación de afectación fetal causada de la anemia que se está produciendo por medio de ecografía doopler en la investigación de VS-ACM. Podemos recurrir a un tratamiento teniendo dos clases de enfoques, la realizada a la madre y aquel que se lleva a cabo al feto. 
El tratamiento materno incluye dos medidas que se van a encargar en la reducción de niveles plasmáticos de anticuerpos anti-D. Estos son la plasmoféresis y la administración de gammaglobulina.

La plasmoféresis implica el recambio plasmático materno con alta concentración de anticuerpos anti-D, los niveles d aloanticuerpos pueden ser removidos hasta en un $75 \%$, lo cual puede seguirse con un rebote después de 6 a 8 semanas aun con la realización de plasmoféresis.

La administración de inmunoglobulina intravenosa puede evitar este rebote y mantener niveles adecuados de IgG. Esto se debe comenzar a las 10 o 12 semanas de gestación, cuando comienza la transferencia de anticuerpos maternos al feto. Dado los efectos adversos de esta medida y su costo, solo debe de administrarse a pacientes con parejas homocigotas para dicho gen $\mathrm{Rh}+\mathrm{y}$ con una historia previa de hydrops.

El tratamiento fetal tiene como objetivo reducir la hemolisis y la anemia. El principal tratamiento es la transfusión intrauterina de sangre Rh (D) negativo, que se realiza en dos modalidades: la transfusión intravenosa mediante cordocentesis y transfusión intraperitoneal.

El objetivo de la transfusión intravenosa mediante cordocentesis es llegar a un hematocrito fetal de 40 a 45\%, realizándose la punción preferentemente en la vena umbilical.

La transfusión intraperitoneal se realiza cuando no es posible realizar a transfusión intravenosa. En esta técnica se administra hematíes en la cavidad peritoneal para ser absorbidos a través de las lagunas linfáticas subdiafragmáticas, sin importar la presencia a ascitis ya que no impide su absorción. 
La conducta a seguir en cuanto a continuar con el parto o interrumpirlo va a depender en que zona se encuentre el feto en el gráfico de Liley. Si se encuentra en la zona baja o media se continuara la gesta hasta llegar a término teniendo un seguimiento exhaustivo. Pero si se encuentra en la zona alta se procede a la interrupción de embarazo dependiendo su maduración; en la cual primero se maduran los pulmones para aumentar su viabilidad postparto, continuando con su interrupción.

\section{Caso clínico.}

En la clínica Kennedy alborada el 17/11/2015 se atendió una paciente secundigesta Rh (D) negativo de 31 años, que curso con amenorrea de 5 semanas, realizándole eco transvaginal que mostro un saco de $3 \mathrm{~cm}$ uniforme, en el examen general. Hematíes $=5130.000, \mathrm{Hg}=15.6$ y hematocrito $=44.70$. Le realizamos test de Coombs indirecto el cual fue negativo.

Como antecedente tenemos que su gesta anterior termino en cesaría y presento tialósis intensa de 1er T y 2do T.

En su 2do control el 2/12/15 se le calcula 6.3 semanas de gestación. Con presencia de tialósis intensa manteniéndola en hidratación + ácido fólico y hierro. Y síntomas de dolor pélvico leve (se le administro progesterona). Se le calculo la fecha de parto dándonos un aproximado el 8-15/07/16.

Se le realizo controles mensuales para ver el desarrollo de la gestación y el cambio del test de Coombs ya que es de vital importancia para prevenir trastornos hemolíticos en el feto. En el 3er control se observó disminución de la tialósis, 4to control se evaluó el 
desarrollo, la observación del hueso nasal y la presencia de movimientos de las 4 extremidades, 6to control se identificó su sexo(masculino), 7mo, 8vo 9no control se realizó el seguimiento de su desarrollo viendo el incremento de peso.

Hasta el 9no control, en la semana 30 gestacional, el test de Coombs indirecto nos da como resultado negativo. Pero se identificó policromatofilia en el examen general en frotis periférico, lo que nos da a relación un inicio de problemas hematológicos. También encontramos ligero edema en piel en el feto. En el doopler los valores estándar están dentro del rango y VSA: $8.21 \mathrm{~cm}$.

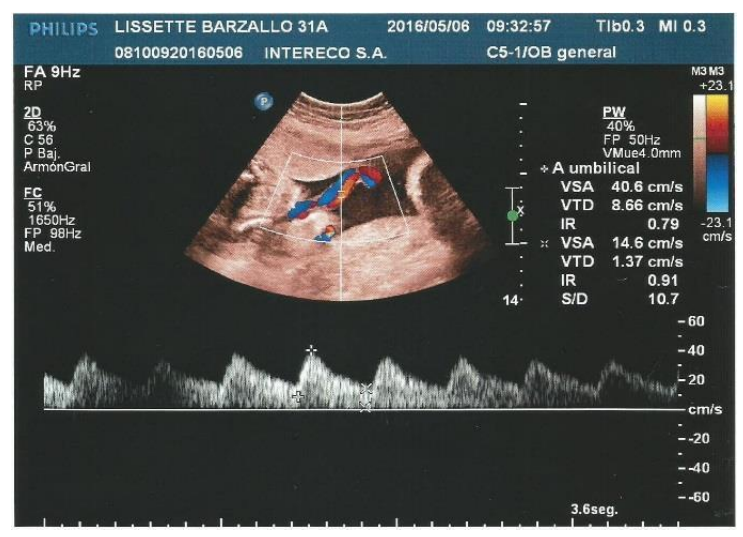

Imagen 1: IR-A.UMB: 0.91

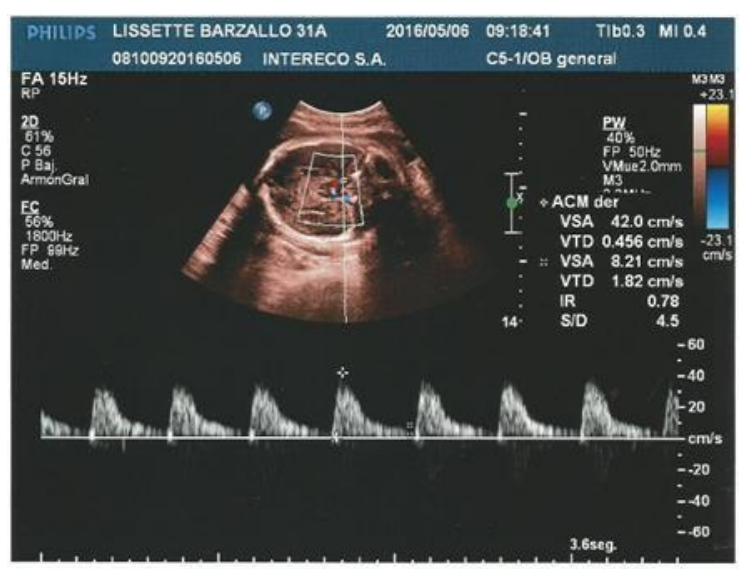

Imagen 2: ACM; IR: 0.78, VSA: 8.21 
En el 10mo control realizado el 31/05/2016 el test de Coombs indirecto nos da como resultado positivo, demostrando que ya ha ocurrido sensibilización y la producción de anticuerpos anti-D, realizamos la titulación para cuantificar y nos dio 1/2048. Se eligió seguir con el mismo tratamiento.

En la 11ava consulta el eco doopler nos revela una IR ACM: 0.75, IR AUMB: 0.78 y VSA: $10.9 \mathrm{~cm}$. Presencia de líquido debajo de piel y edematisada, intestino ligeramente edematoso e hidrocele. El siguiente test de Coombs indirecto realizado sigue igual al anterior con una cuantificación de 1/2048. Se procede a interconsultar con equipo médico pediátrico y hematóloga.

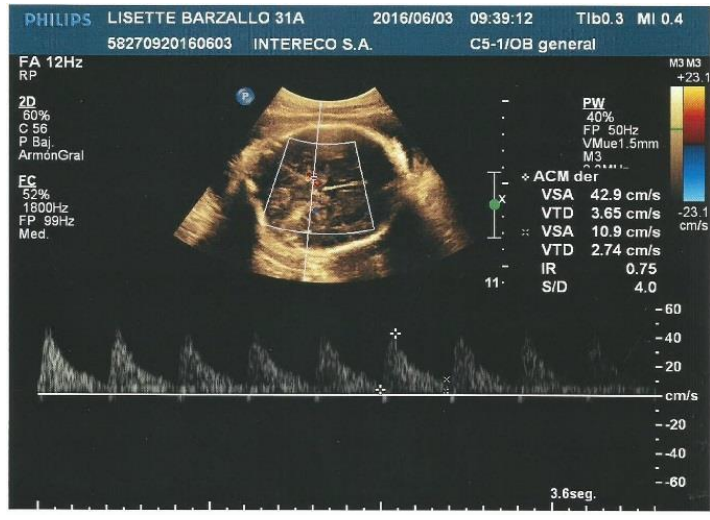

Imagen 3: IR ACM: 0.75 y VSA: $10.9 \mathrm{~cm}$

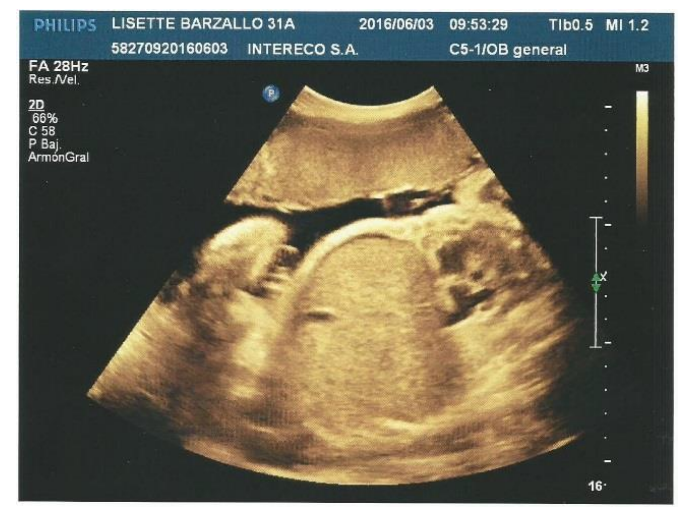

Imagen 4: Presencia de piel edematisada 
En la 12ava consulta realizada 5 días después el teste de Coombs indirecto no cambia, existe policromatofilia más marcada y se procede a administrar corticosteroides para ayudar a la maduración pulmonar y disminuir la hemolisis.

La 13ava visita se realiza 3 días después para valorar la viabilidad del embarazo donde el examen doopler nos da un incremento en los marcadores de IR ACM: 0.79, IR AUMB: 0.81 y VSA: $13.2 \mathrm{~cm}$. También se observa hidrocele, pared abdominal con edema, cotiledones con características edematisadas y GDMP: 2/3.

La edad gestaciones estaba en las 35.1 semanas. Dándonos una buena oportunidad de sobrevida por lo cual se procedió a extraerlo realizándose cesaría segmentaria, y tenemos producto vivo con L.A amarillento y observando neonato ictérico. Se entrega a pediatra el cual lo pasa a cama con fototerapia. Posteriormente se le realiza dos exanguineotransfusiones con espacio de tiempo que dio buena respuesta y el niño actualmente goza de buena salud..

La intervención quirúrgica se dio en el HOSPITAL OMNIHOSPITAL

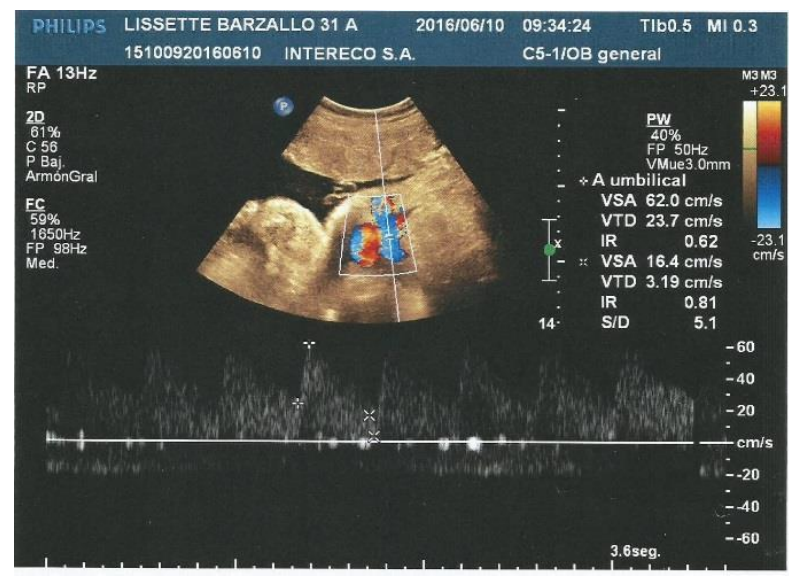

Imagen 5: IR A.UMB: 0.81 


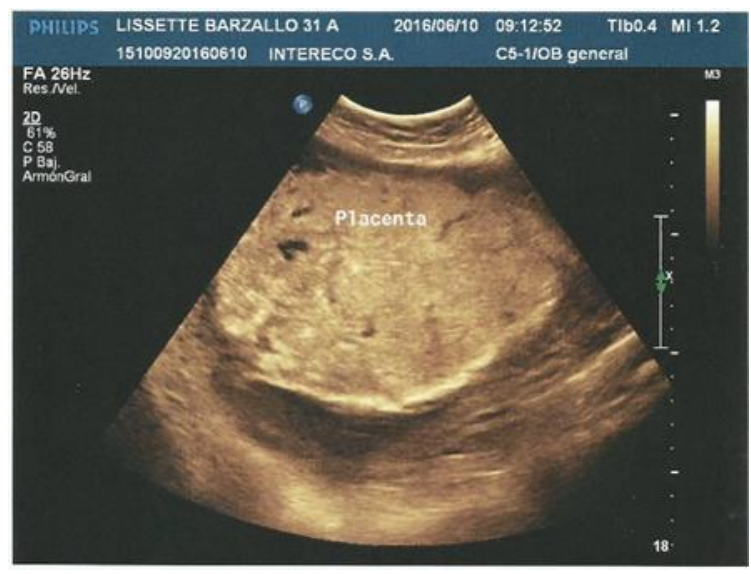

Imagen 6: Placenta edematosa con presencia de cotiledones.

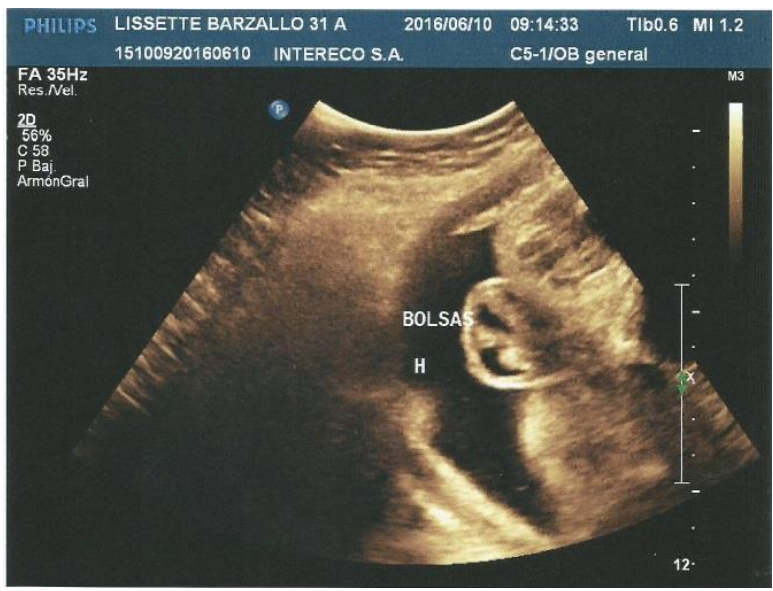

Imagen 7: Hidrocele.

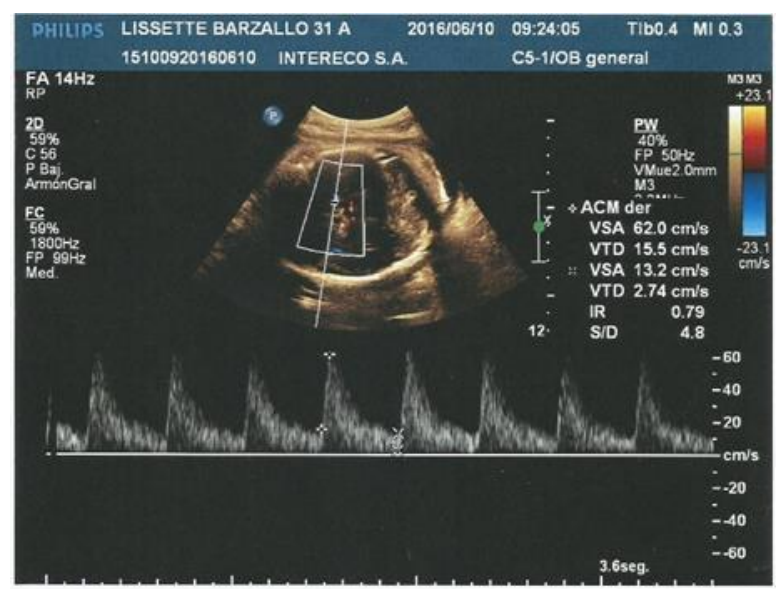

Imagen 8: ACM; IR: 0.79, VSA: 13.2 


\section{Conclusiones.}

El presente caso, se presenta con la finalidad de que podamos disminuir la incidencia de morbimortalidad que ocasiona esta patología de incompatibilidad Rh. Que la nuevas técnicas de laboratorio e imágenes, nos permitan llevar mayor control y actuar adecuadamente con el binomio madre hijo.

Que la administración anti D profilácticamente se aplique en los seguimientos y etapas adecuadas y con mucha más razón en los siguientes embarazos en los que no haya existido sensibilización.

Que tanto la cuantificación en el laboratorio, del test de Coombs indirecto y la determinación de la onda VSA de ACM en su mayor pico en un eco doopler nos valla dando los estados de anemia y hemolisis que pudieran estar ocurriendo en el feto y en con conjunto con la valoración ecográfica fetal; ver el secuestro de líquidos, como el edema de piel, hidroceles, etc. Nos permita todo estos exámenes en conjunto se indicativos para de acuerdo a su evolución determinar la extracción fetal adecuada; reduciendo al mínimo los exámenes invasivos que aumentan el riesgo de morbimortalidad fetal, de tal manera que el binomio madre-hijo lleguen a un buen término.

\section{Bibliografía.}

1. Insunza F Alvaro, Behnke G Ernesto, Carrillo T Jorge. Enfermedad hemolítica perinatal: manejo de la embarazada RhD negativo. Rev. chil. obstet. ginecol. 2011; 76(3): 188-206.

2. Diamond LK, Blackfan KD, Batty JM. Erythroblastosis fetalis and its association with universal edema of the fetus, icterus gravis neonatorum and anemia of the newborn. J Pediatr 1932;1:512-0.

3. Levine P. The role of isoimmunization in transfusion accidents in pregnancy and erythroblastosis fetalis. Am J Obstet Gynecol 1941;42:165-0. 
4. Arévalo MJ, Bellazzi MV, Zanazzi DD, Arévalo JC. Revista de Posgrado de la Cátedra de Medicina. 2009; 195(1): 17-22.

5. Kumpel B. Lessons learnt from many years of experience using anti-D in humans for prevention of RhD immunization and haemolytic disease of the fetus and newborn. Clinical and Experimental Immunol. 2008; 154(1): 1-5.

6. Augustson BM, Fong EA, Grey DE, Davies JI , Erber WN. Postpartum anti-D: can we safely reduce the dose?. MJA. 2006; 184(1): 611-613.

7. Swarz R, Fescina R, Duverges C. Obstetricia 6 ${ }^{\mathrm{a}}$ Ed. Buenos Aires: Editorial El Ateneo; 2006: 415-427. 